\title{
Neonatal Pneumocephalus Secondary to Acinetobacter Meningitis
}

\author{
Richie Dalai ${ }^{1} \cdot$ Mayank Priyadarshi ${ }^{1,2} \cdot$ Ankit Verma $^{1} \cdot$ Priyanka Naranje $^{3}$
}

Received: 2 February 2021 / Accepted: 25 February 2021 / Published online: 15 March 2021

(C) Dr. K C Chaudhuri Foundation 2021

To the Editor: A female term neonate presented to the emergency department on day 6 of life, limp and apneic. She was immediately resuscitated, mechanically ventilated, rewarmed (initial axillary temperature $31{ }^{\circ} \mathrm{C}$ ) and managed with fluid boluses and inotropes for decompensated shock. She had history of lethargy and poor feeding since day 2 of life. She was managed as sepsis at a health facility for past 3 days, where her blood culture grew methicillin-resistant Staphylococcus aureus (MRSA), cerebrospinal fluid (CSF) was sterile. The neonate was referred from that facility for worsening sensorium.

At our center, she continued receiving supportive treatment and antibiotics (vancomycin and piperacillin-tazobactum). Lumbar puncture was deferred due to severe hemodynamic instability. Computed tomography (CT) imaging of brain (noncontrast due to acute kidney injury) was suggestive of diffuse cerebral edema with pneumocephalus in the posterior horn of right lateral ventricle extending to parenchyma without causing any midline shift. Neurosurgical intervention was deferred owing to hemodynamic instability and poor outcome. Despite ongoing therapies, she expired on day 11 of life. Her blood and CSF cultures (frank pus on postmortem tap) later grew Escherichia coli and Acinetobacter baumanii, respectively (both organisms resistant to third generation cephalosporins and carbapenems, sensitive to tigecycline and polymyxin B), possibly explaining refractoriness to instituted antimicrobials.

Ankit Verma

ankitvmmc@yahoo.co.in

1 Department of Pediatrics, All India Institute of Medical Sciences, New Delhi 110029, India

2 Present address: Department of Neonatology, All India Institute of Medical Sciences, Rishikesh, Uttarakhand, India

3 Department of Radiodiagnosis, All India Institute of Medical Sciences, New Delhi, India
Though reported in adults, this is the first case in neonates of spontaneous pneumocephalus with Acinetobacter meningitis [1]. Pneumocephalus is defined as the presence of air within the cranial cavity. Common causes include trauma and neurosurgical interventions but can occur with central nervous system (CNS) infections (due to gas production with bacterial proliferation) [2]. It is usually self-limiting but, in few cases, can progress to cause tension pneumocephalus, resulting in poor neurological outcome [3]. Majority of the cases resolve with conservative management, while surgery is reserved for those who have associated raised intracranial pressure, recurrent episodes, and persistence beyond $1 \mathrm{wk}$ [4].

\section{Declarations}

Consent to Publish The legal guardian has consented regarding publishing the data.

Conflict of Interest None.

\section{References}

1. Moiyadi AV, Shetty P, Biswas S. Fulminant post-craniotomy wound infection and meningitis with pneumocephalus caused by acinetobacter baumannii: an unusual presentation. J Neurosci Rural Pract. 2013;4:S136-7.

2. Shenoi AN, Shane AL, Fortenberry JD, Abramowsky C, Kamat P. Spontaneous pneumocephalus in vertically acquired, late-onset neonatal citrobacter meningitis. J Pediatr. 2013;163(6):1791.

3. Sedaghatian MR, Ramachandran P, Rashid N. Diffuse pneumocephalus caused by neonatal Enterobacter cloacae meningitis. Arch Dis Child Fetal Neonatal Ed. 2004;89:F324.

4. Pooboni SK, Mathur SK, Dux A, Hewertson J, Nichani S. Pneumocephalus in neonatal meningitis: diffuse, necrotizing meningo-encephalitis in Citrobacter meningitis presenting with pneumatosis oculi and pneumocephalus. Pediatr Crit Care Med. 2004;5(4):393-5.

Publisher's Note Springer Nature remains neutral with regard to jurisdictional claims in published maps and institutional affiliations. 